\title{
Non Mechanical (Mezic) Type Forces in the Foundations of Quantum Mechanics
}

\author{
Č. Šimáně
}

\begin{abstract}
Many authors have attempted to derive the fundamental equations of quantum mechanics from classical hydrodynamics. In the present contribution we presume that the continuous, electrically charged material substance moves simultaneously under the influence of the electric field and at the same time undergoes a diffusion process. This assumption leads to the appearance of non-mechanical (mezic) type forces responsible for inner sources of matter (positive or negative), similar to those whose existence is supposed to exist in relativistic hydrodynamics. We obtained a non-linear differential equation, convertible by linearization to a form coinciding with the Schrödinger equation, as a condition for the establishment of the same steady states with discrete energies.
\end{abstract}

Keywords: hydrodynamics, quantum mechanics, mezic (non-mechanical) forces.

\section{Introduction}

The discovery of quantum mechanics signified a revolution in the physical image of our world. However, the price paid for the general acceptance of quantum mechanics was very high. One had to sacrifice many fundamental principles of classical mechanics, among them especially its determinism, and to accept controversial uncertainty relations. The constant $\hbar$ discovered by Planck together with Einstein's relations $E=h \nu$ and $\vec{p}=\vec{k} \nu$ between the corpuscular and wave aspects of matter were the starting points for the discovery of quantum mechanics by Schrödinger, Louis de Broglie, Born, Heisenberg and Dirac. Many physicists tried to overspan the profound abyss between classical and quantum mechanics, proposing various models leading to the fundamental equations of quantum mechanics [1] . This work is in some way a continuation of the author's earlier article on this subject [2].

\section{From classical to quantum mechanics}

The Schrödinger equation for a spinless particle in spherical S states of the hydrogen atom has been chosen to demonstrate that the assumption of the existence of non-mechanical (mezic) forces and diffusion of electrically charged continuous electron matter in the field of electric potential are sufficient to deduce its formal equivalent from classical hydrodynamics. Because one can hardly reconcile the continuous hydrodynamical system with the electrons as particles in the planetary model of the atom, the concept of particles is the first that had to be sacrificed. Instead of this, one has to replace them by a cloud of charged electron matter around the nucleus. Further, one has to propose some mechanism which could lead to the existence of discrete time independent states of the system. Inspiration comes from the paper by Nelson [3], who used statistical mechanics to derive equations that led to discrete states in planetary atomic systems. His basic idea was that the electron simultaneously executes two kinds of motions. One, which is the classical kind, with velocity $\vec{v}$ (he refers to it as the flow velocity) in the electrostatic field of the nucleus, and a certain type of Brownian motion resulting in a motion with osmotic velocity $\vec{u}$ obeying the diffusion law

$$
\mu \vec{u}=-D \nabla \mu
$$

which leads to relations

$$
\begin{aligned}
& \vec{u}=-D \frac{\nabla \mu}{\mu}, \quad \nabla \mu=-D^{-1} \mu \vec{u}, \\
& u^{2}=D^{2} \frac{(\nabla \mu)^{2}}{\mu^{2}}, \quad \operatorname{div}(\mu \vec{u})=-D \Delta \mu
\end{aligned}
$$

Inspired by Nelson, we constructed a heuristic Lagrangean $L$ for the motion of a volume element $\delta V$ containing $m \mu \delta V$ electron matter ( $m$ is the electron rest mass, $\mu$ the distribution function normalized to unity, $e \mu$ the electric charge density)

$$
L=\int_{\delta V} \mu\left(m \frac{v^{2}+u^{2}}{2}-\mathrm{e} \varphi(\vec{x})\right) \mathrm{d} V
$$

as a sum consisting of two Lagrangeans in which the integrand is the sum of the densities of the kinetic potentials corresponding to the motions with uncorrelated velocities $\vec{v}$ and $\vec{u}(\operatorname{rot} \vec{v}=\operatorname{rot} \vec{u}=0)$ and the 
density of the potential energy e $\mu \varphi$. For infinitely small $\delta V$ the Lagrangean is replaceable by

$$
L=m \mu\left[\frac{v^{2}+u^{2}}{2}-\mathrm{e}\left(\varphi-\varphi_{0}\right)\right] \delta V
$$

From the general formula

$$
\frac{\mathrm{d}}{\mathrm{d} t} \frac{\partial L}{\mathrm{~d} \dot{q}_{i}}-\frac{\partial L}{\mathrm{~d} q_{i}}=0
$$

we get the equations of motion of the volume element

$$
\begin{aligned}
& \frac{\mathrm{d}}{\mathrm{d} t}[\mu(\vec{v}+\vec{u}) \delta V]- \\
& \nabla\left[\mu\left(\frac{\left(v^{2}+u^{2}\right)}{2}-\frac{\mathrm{e}}{m}\left(\varphi-\varphi_{0}\right)\right)\right] m \delta V=0,
\end{aligned}
$$

The $\varphi_{0}$ is for the moment an arbitrary constant potential, which disappears in the potential gradient.

The first member on the left side of (5) is the inertial force acting on the volume element $\delta V$ by acceleration. The second member represents the external force densities.

$$
\begin{aligned}
& {\left[-\mathrm{e} \frac{\mathrm{e}}{m} \mu \nabla \varphi+\right.} \\
& \left.\left(\frac{\left(v^{2}+u^{2}\right)}{2}-\frac{\mathrm{e}}{m}\left(\varphi-\varphi_{0}\right)\right) \nabla \mu\right] m \delta V
\end{aligned}
$$

The first member of (6) is the classical electrical force density.

$$
-\frac{\mathrm{e}}{m} \mu \nabla \varphi
$$

The second member is interesting. If $\nabla \mu$ is substituted from (1b), then this force density becomes

$$
\vec{u} D^{-1} \mu\left(\frac{v^{2}+u^{2}}{2}-\frac{\mathrm{e}}{m}\left(\varphi-\varphi_{0}\right)\right)=\vec{u} q,
$$

a product of the velocity $\vec{u}$ and the sum of the densities of the kinetic and electrical potentials multiplied by $D^{-1}$. Setting $D=\hbar /(2 m)$ with dimension $\mathrm{m}^{2} \mathrm{~s}^{-1}$, $q$ gets dimension $\mathrm{kg} \mathrm{s}^{-1}$ and may be interpreted as the change of matter content in a volume element in the time unit, i.e, as the density of an internal source of matter.

Then, the equations of motion can be explicitly written in the form

$$
\begin{aligned}
& {\left[\mu \frac{\mathrm{d} \vec{v}}{\mathrm{~d} t}+\mu \frac{\mathrm{d} \vec{u}}{\mathrm{~d} t} \delta V+(\vec{v}+\vec{u}) \frac{\mathrm{d} \mu}{\mathrm{d} t} \delta V+\right.} \\
& \left.\mu(\vec{v}+\vec{u}) \frac{(\delta V)}{\mathrm{d} t}+\frac{\mathrm{e}}{m} \mu \nabla \varphi \delta V-\vec{u} q\right] m \delta V=0
\end{aligned}
$$

The time derivative of $\delta V$ in the fourth member of (8) must be treated as the sum of the derivatives at $\vec{v}$ and $\vec{u}$ constant

$$
\begin{aligned}
& \mu(\vec{v}+\vec{u}) \frac{(\delta V)}{\mathrm{d} t}= \\
& \mu(\vec{v}+\vec{u})\left[\operatorname{div}_{\vec{u}}(\vec{v}+\vec{u})+\operatorname{div}_{\vec{v}}(\vec{v}+\vec{u})\right] \delta V= \\
& \mu \vec{v} \operatorname{div} \vec{v} \delta V+\mu u \operatorname{div} \vec{u} \delta V
\end{aligned}
$$

Then the equations of motion of the unit volume element take the form

$$
\begin{aligned}
& \mu \frac{\mathrm{d} \vec{v}}{\mathrm{~d} t}+\mu \frac{\mathrm{d} \vec{u}}{\mathrm{~d} t}+\vec{v}\left[\left(\frac{\partial \mu}{\partial t}\right)_{\vec{u}}+\vec{v} \nabla \mu\right]+ \\
& \mu \vec{v} \operatorname{div} \vec{v}+\vec{u}\left[\left(\frac{\partial \mu}{\partial t}\right)_{\vec{v}}+\vec{u} \nabla \mu\right]+ \\
& \mu \vec{u} \operatorname{div} \vec{u}+\frac{\mathrm{e}}{m} \mu \nabla \varphi+\vec{u} q=0
\end{aligned}
$$

or by putting together the third and the fourth, and the fifth, sixth and the last member members in (10)

$$
\begin{aligned}
& \mu \frac{\mathrm{d} \vec{v}}{\mathrm{~d} t}+\vec{v}\left[\left(\frac{\partial \mu}{\partial t}\right)_{\vec{u}}+\operatorname{div}(\mu \vec{v})\right]+\frac{\mathrm{e}}{m} \mu \nabla \varphi+ \\
& \mu \frac{\mathrm{d} \vec{u}}{\mathrm{~d} t}+\vec{u}\left[\left(\frac{\partial \mu}{\partial t}\right)_{\vec{v}}+\operatorname{div}(\mu \vec{u})\right]+\vec{u} q=0
\end{aligned}
$$

Here again the partial time derivatives must be taken at $\vec{v}$ and $\vec{u}$ constant.

The sum of the first and the third member in (11) is the equation of motion

$$
\mu \frac{\mathrm{d} \vec{v}}{\mathrm{~d} t}+\frac{\mathrm{e}}{m} \mu \nabla \varphi=0
$$

and the expression in the square brackets in the second member in (11) the equation of continuity

$$
\left(\frac{\partial \mu}{\partial t}\right)_{\vec{u}}+\operatorname{div}(\mu \vec{v})=0
$$

in the motion with the velocity $\vec{v}$.

The remaining members in (11) represent the equation of motion with velocity $\vec{u}$, which is according to (1) a function of $\mu$. The last two members in (11) can be put together, so that the equation of motion becomes

$$
\mu \frac{\mathrm{d} \vec{u}}{\mathrm{~d} t}+\vec{u}\left[\left(\frac{\partial \mu}{\partial t}\right)_{\vec{v}}+\operatorname{div}(\mu \vec{u})+q\right]=0
$$

The expression in square brackets in the second member in (12b) may be interpreted as an equation of continuity with internal sources of matter $q$ expressed by (7b)

$$
\begin{aligned}
& \left(\frac{\partial \mu}{\partial t}\right)_{\vec{v}}+\operatorname{div}(\mu \vec{u})- \\
& D^{-1} \mu\left(\frac{v^{2}+u^{2}}{2}-\frac{\mathrm{e}}{m}\left(\varphi-\varphi_{0}\right)\right)=0
\end{aligned}
$$




\section{$3 \quad$ Steady states}

From now on, we will be interested only in steady, time independent solutions of the equations of motion and of the continuity equations. Therefore, we shall omit all partial time derivatives.

Equations (12a) and (13a) concern the classical motion of matter in the electrostatic field of the nucleus, for which the equation of continuity (13a) reduces to the second member or to

$$
\vec{v} \nabla \mu+\mu \operatorname{div} \vec{v}=0
$$

The gradient of density $\mu$ may take any value between minus and plus infinity, so that equation (14) can be satisfied only for $\vec{v}$ equal identically to zero everywhere. This of course is inconsistent with (12a), unless another electric force density compensates the force density in the electrostatic field of the nucleus. The only electrical charges present that could create the compensating electric field are the charges of the electron cloud.

Supposing spherical symmetry of the electrical charge, the total flux of the electrical induction through the surface of the sphere of radius $R$ equals the charge within this sphere

$$
4 \pi R^{2} \vec{E}=-\mathrm{e} 4 \pi \int_{\rho=0}^{R} \mu(\rho) \rho^{2} \mathrm{~d} \rho
$$

Because the charges outside the sphere do not contribute to the intensity, one can extend the integration on the whole space and thus, owing to normalization of the distribution function, the field intensity

$$
\vec{E}=-\mathrm{e} \frac{4 \pi}{4 \pi R^{2}} \int_{\rho=0}^{\infty} \mu(\rho) \rho^{2} \mathrm{~d} \rho=\frac{-\mathrm{e}}{R^{2}}
$$

The resulting electrical intensity from the positive charge of the proton and the negative charge of the electron cloud

$$
E_{n}+E_{e}=\frac{\mathrm{e}}{R^{2}}-\frac{\mathrm{e}}{R^{2}}=0
$$

Now, let us pay attention to the process with osmotic velocity $\vec{u}$, to which equations (12b) and (13b) pertain. Equation (12b) of motion with osmotic velocity has the form

$$
\vec{u} \mu \operatorname{div} \bar{u}+\vec{u}[\operatorname{div}(\mu \vec{u})+q]=0
$$

If the equation of continuity (13b) is valid, then the second member in (18) equals zero and the equation of motion reduces to

$$
\vec{u} \operatorname{div} \bar{u}=\frac{\mathrm{d} \vec{u}}{\mathrm{~d} t}=0 \quad\left(\frac{\partial \vec{u}}{\partial t}=0\right),
$$

in agreement with the characteristic property of the mezic forces, which do not accelerate the material substance, so that its spatial distribution continues to be time independent.

After replacing in (13b) from $(2) u^{2}$ by $D^{2} \frac{(\nabla \mu)^{2}}{\mu^{2}}$ and $\operatorname{div}(\mu \vec{u})$ by $-D \Delta \mu$, we get the equation of continuity in the form

$-D \Delta \mu+D^{-1} \mu\left(\frac{v^{2}}{2}+\frac{D^{2}(\nabla \mu)^{2}}{2 \mu^{2}}-\mathrm{e}\left(\varphi-\varphi_{0}\right)\right)=0$,

which is then the condition for a time independent, steady state of the substance in the volume element at a given point of the cloud. Simultaneously, equation (14) must also be satisfied, which - as shown above - is possible only for $\vec{v}$ equal identically to zero. Therefore, the final condition for a steady, time independent state has the form

$$
-D \Delta \mu+D^{-1} \mu\left(\frac{D^{2}(\nabla \mu)^{2}}{2 \mu^{2}}-\mathrm{e}\left(\varphi-\varphi_{0}\right)\right)=0
$$

The solution $\mu\left(\vec{x}, \mathrm{e} \varphi_{0}\right)$ of (14) depends on the value of the constant $\mathrm{e} \varphi_{0}$. If in all points in space the solution fulfils (14) for the same value of $\mathrm{e} \varphi_{0}$, then equation (21) represents the condition for a steady state of the whole cloud and may be interpreted in the following way:

The steady, time independent state of the cloud is reached when in each one of its volume elements the outflow (inflow) of the matter through its surface is just compensated by the internal positive (negative) sources of matter evoked by non-mechanical (mezic) type forces.

Substituting $\mu=R^{2}$ in the non linear equation (21), one obtains a linear equation

$$
\left(\frac{\hbar^{2}}{2 m} \Delta+\mathrm{e} \varphi\right) R=\mathrm{e} \varphi_{0} R \equiv E R
$$

Equation (22), derived from a hydrodynamic model, is in fact sufficient to bring order into most of the experimental spectroscopic data of discrete energy states. It does not resemble a wave equation. It is asymmetric: on the left side we have an operator, and on the right side a simple algebraic expression. Replacing this expression by an operator of the form $-i \hbar \partial / \partial t$ acting on a exponential function

$$
\psi=R \exp \left(-i \frac{E}{\hbar} t\right)
$$

we obtain the same equation (18), but with operators on both sides

$$
H \psi=-i \hbar \frac{\partial}{\partial t} \psi
$$

where $\psi$ can rightly be declared as a wave function.

The author would like to remark, that one gets the same results if the velocity $\vec{u}$ is taken from the beginning as pure imaginary. Then (11) is obtained as a 
complex equation and separating the real and imaginary parts one immediately gets equations $(12 \mathrm{a}, \mathrm{b})$ and $(13 \mathrm{a}, \mathrm{b})$.

Before finishing this chapter, the author would like to mention the historical paper by D. Bohm [4] concerning the so-called hidden variables of quantum theory.

Bohm supposed the function $\psi=R \exp (-i S / \hbar)$, ( $R$ and $S$ are real functions of time and coordinates), as the solution of the Schrödinger equation

$$
i \hbar \partial \psi / \partial t=-\left(\hbar^{2} / 2 m\right) \nabla^{2} \psi+V(\vec{x}) \psi,
$$

Substituting $R$ by $\mu^{1 / 2}$, putting $\frac{\partial S}{\partial t}=E$ and $\vec{v}=$ $\frac{\nabla S}{m}$, he obtained from (24) two equations

$$
\begin{array}{r}
\left(\frac{\partial \mu}{\partial t}\right)+\nabla\left(\mu \frac{\nabla S}{m}\right)=0 \\
\frac{\partial S}{\partial t}+\frac{(\nabla S)^{2}}{2 m}-\mathrm{e} \mu \varphi-\frac{\hbar^{2}}{4 m}\left[\frac{\Delta \mu}{\mu}-\frac{(\nabla \mu)^{2}}{2 \mu^{2}}\right]=0
\end{array}
$$

Oriented on the planetary atomic model, Bohm interpreted (26b) as a Jacobi-Hamilton equation for the motion of a particle in the field of classical electric potential and in the field of a quantum potential expressed by the last member on the left side of (26b). However, this equation, multiplied by $\mu / D$, with $\nabla S / m$ replaced by $\vec{v}$, and $\frac{\mu}{D} \frac{\partial S}{\partial t}$ by $\left(\frac{\partial \mu}{\partial t}\right)_{\vec{v}}$ and with the use of $(1 \mathrm{~b})$ can be transformed into equation (13b) with an entirely different interpretation.

\section{Discussion}

A necessary condition for the existence of steady, time independent spatial density distribution and fluxes of the electronic substance in an electrically charged material continuum is the validity of the equation of continuity with internal sources or sinks of matter, which compensate the outflows or inflows of matter in each of the volume elements of the object through its surface.

The basic prepositions necessary for the derivation of this equation of continuity reducible formally to a form of the Schrödinger equation were that

- the electron in the bound state does not exist as a particle and forms a cloud of continuous electrically charged electron substance around the nucleus, bearing the total electrical charge, whose spatial distribution is the same as that of the electron substance.

- there exist two kinds of uncorrelated motions of the continuum, one with flow velocity $\vec{v}$ and the other with osmotic velocity $\vec{u}$, the latter obeying the diffusion law.

- the continuous electron substance moves in the electric field of the nucleus and in the field of its own electrical charge and at the same time diffuses opposite to the density gradient.

- inner sources or sinks of matter due to the action of non-mechanical (mezic) type forces exist in the continuous electron substance.

- in the steady time independent case, the flow velocity is zero and the intensity of the electrical field of the nucleus is compensated by the electron field of the electron cloud.

- the possibility of reducing the nonlinear equations of continuity to the linear Schrödinger equation offers a practical means for solving them.

It is worth noting that continuous distribution of the electron matter around the nucleus was strongly defended by Schrödinger in his disputes with Born [5].

In relativistic hydromechanics, the existence of inner sources of matter is attributed to the action of forces of non-mechanical (mezic) character [6]. Nonrelativistic hydromechanics does not calculate with their existence. As such, they have never been observed, because they vanish in steady states and one can conclude their existence only indirectly from the fact that with their aid one can get the same steady time independent states as from the corresponding Schrödinger equation. Nevertheless, the non-mechanical forces should manifest their existence in non-stationary processes during so-called jumps from one state to another. The violation of the continuity equation in the steady state by external perturbation cancels this state and starts the transition from a steady state to a time dependent state. This transition process may end in reestablishment of the initial steady state or in a transition to some new state with different energy. Without an exact mathematical description of the transition process, which is very rapid and which manifests itself as a jump, one can only predict the probability of the transitions to the end states. The situation could change, once one will be able to follow theoretically the transition process in time, in which the non-mechanical forces will play an important role.

A very serious historical difficulty was mentioned already by Madelung [7] in his attempt to deduce a hydrodynamical model from the Schrödinger equation, namely that one has to assume that the electrically charged volume elements of the same electron cloud do not mutually interact. In the planetary model, this difficulty disappears, as the inner electric field is concentrated in the point-like particle. We suppose that the inner electrical intensity in the electron cloud compensates just the electrical intensity in the electric field of the nucleus, which allows the flow intensity in the discrete, time independent state to be obtained equal to zero.

We have no idea how to describe the process by which the continuous electron cloud takes the form 
and properties of a free particle. The dual wave particle character, manifested mainly in the vicinity of the rest energy of the free particle and depending on the type of the experiment, might be explained as fluctuations between the continuous and particle states of the electron, due to small perturbations of the continuity equations provoked by weak external fields, by their time dependence and boundary conditions in various types of experiments.

\section{Conclusion}

The hydrodynamical equations of motion deduced above, which are based on Newtonian mechanics and include non-mechanical (mezic) forces combined with the diffusion of the charged electronic substance in the field of an electric potential, may be considered in only a limited sense equivalent to quantum mechanics based on the discoveries by Schrödinger, Louis de Broglie, Born, Heisenberg, Dirac, covering a much broader range of experimentally observed quantum phenomena. It is too early to draw general conclusions from the one isolated special case treated above. Nevertheless, one should not omit to pay attention to the possible role of mezic forces in quantum mechanics.

\section{References}

[1] Jammer, M.: The Philosophy of Quantum Mechanics, J. Wiley \& Sons, New York, 1974.
[2] Šimáně, C.: Discrete states of continuous electrically charged matter. Concepts of Physics, Vol. V, No. 3, 2008, 499-512.

[3] Nelson, E.: Derivation of the Schrödinger equation from Newtonian mechanics, Phys. Rev. 50, 1966, 1079-1085.

[4] Bohm, D.: A suggested interpretation of the quantum theory in terms of "hidden variables "I, Phys. Rev. 85, 1952, 166-179.

[5] Born, M.: The conceptual situation in Physics and the prospects of its future development, Proc. Phys. Soc., A, Vol. LXVI,1953, 501-513.

[6] Möller, C.: The theory of relativity, second edition, Clarendon Press Oxford 1972, Ruskoe izdanie, Moskva Atomizdat 1975, pp. 105-108;

Votruba, V.: Základy speciální teorie relativity, Academia Press, Prag 1969, pp. 307-313.

[7] Madelung, E.: Quantentheorie in hydrodynamischer form, Zeitschr. für Physik, 40, 1926, 166.

Prof. Dipl. Ing. Čestmír Šimáně, DrSc.

Phone: +420284819279

E-mail: csimane@centrum.cz

Nuclear Physics Institute AS CR

Prof. Emeritus of the Czech Technical University in Prague

Home adress:

U svobodárny 9, 19000 Praha 9, Czech Republic 